\title{
INFLUENCE DES HORMONES GONADOTROPES SUR LA DURÉE DES PROCESSUS SPERMATOGÉNÉTIQUES CHEZ LE RAT
}

\author{
J. DESCLIN et R. ORTAVANT \\ avec la collaboration technique de Marie-Madeleine de Reviers. \\ Laboratoire d'Histologie, Universite' Libre, Bruxelles (Belgique); \\ Laboratoire de Physiologie de la Reproduction, Centre national de Recherches zootechniques, \\ Jouy-en-Josas, Seine et Oise
}

\section{SOMMAIRE}

L'influence de l'hypophysectomie et de l'injection d'hormones gonadotropes, sur les durées de la prophase méiotique et de la spermiogenèse a été étudiée chez des Rats mâles adultes après injection de thymidine tritiée.

Après l'hypophysectomie, l'injection de différentes doses (i à c 760 ug de standard Armour) d'hormone folliculostimulante ou d'hormone lutéinisante permet d'obtenir des niveaux d'activité spermatogénétique variant de I $\grave{a}$ I 7 . Il se produit en effet une dégénérescence plus ou moins intense des cellules germinales soit au cours de la prophase méiotique, soit au cours de la spermiogenèse.

Cependant ni la durée des processus spermatogénétiçues, ni la synchronisation du développement des spermatocytes primaires et des spermatides n'ont été perturbées. Les hormones gonadotropes ne modifient le rendement spermatogénétique qu'en agissant sur les phénomènes de dégénérescence.

Des études récentes ont montré que la vitesse du déroulement de la spermatogenèse est variable d'une espèce à l'autre. Ainsi, la durée du cycle de l'épithélium séminifère est de 8 jours environ chez le Verrat, io jours chez le Bélier, I4 jours chez le Taureau (ORTAVANT et al., Ig6I) et I6 jours chez l'Homme (HELLER et CLERMONT, I962). On peut penser que cette variabilité explique en partie la différence de production spermatogénétique chez ces espèces, la production la plus forte se rencontrant dans 1'espèce où la vitesse de déroulement de la spermatogenèse est la plus grande.

Au cours de recherches entreprises par l'un d'entre nous chez le Bélier, diverses conditions expérimentales provoquant des niveaux d'activité spermatogénétique variables n'influencèrent pas la durée du cycle de l'épithélium séminifère (ORTAvanT, 
I958). De même chez le Rat, HARVEY et CI,ERMont (I962) n'ont pas rétussi à modifier la durée de la prophase méiotique par l'hypophysectomie, l'injection de propionate de testostérone ou d'hormone chorionique humaine. La durée de la spermatogenèse semble donc constante dans une espèce. De nombreux travaux ayant cependant conclu à la stimulation et à 1'accélération de 1'activité spermatogénétique par les hormones gonadotropes hypophysaires folliculostimulante et interstitielle stimulante (GaArenstroom et De JoNGH, I946) Greep et al., I936; Simpson et al., I946, I95I ; RANDOLPH et al., I959, Woods et Simpsor, r96r, etc.), il était important de vérifier si ces hormones pouvaient avoir une action sur la durée de certains phénomènes spermatogénétiques : prophase méiotique, spermiogenèse. Nous avons tenté cette vérification chez le Rat.

\section{MATÉRIEI, E'T MÉTHODES}

\section{Les animaux et les traitements}

Nous avons utilisé 62 rats mâles de race Wistar âgés de 3 mois cnviron au début de l'expérience. 53 de ces animaux ont été hypophysectomisés, les autres étant conservés conme témoins. Deux jours après, tous les rats, normaux et opérés, ont reçu une injection intrapéritonéale de thymidine tritiée $(3 \mathrm{C} / \mathrm{mM})$ à la dose de $0,8-1,0 \mu \mathrm{C} / \mathrm{g}$ de poids vif. A partir de ce jour et pendant des périodes de 14 on 24 jours, 44 rats hypophysectomisés ont reçu des injections sous-cutanées bi-quotidiennes d'hormone folliculostimulante (FSII) ou interstitielle stimulante (LH) Armour. Un dosage de contaminants a montré que FSH contenait i p. Ioo d'activité ICSH dosé par la diminution d'acide ascorbique ovarien (PARLow, 1958, modifié par P'ELLETIER, 1963), et que ICSII contenait un taux élevé d'activité FSII (dosé selon STEELMan et l'ohley, I953). Différentes doses de ces hormones soit séparées, soit associées, ont été administrées.

Les animaux ont été abattus 8 heures après la fin du traitement. Au même moment, des rats normaux et des rats hypophysectomisés n'ayant pas reçu d'injections ont été sacrifiés pour servir de témoins. Certaines difficultés expérimentales ne nous ont pas permis d'avoir un nombre constant d'animaux dans chaque lot.

Lors de l'abattage, les testicules et les glandes annexes ont été pesés puis fixés dans le BouinHollande-sublimé (nélange de Bouin-Hollande dans lequel l'acide acétique est remplacé par Io p. Ioo d'unc solution saturéc de sublimé.)

\section{Techniques histologiques et autoradiographiques}

Après déparaffinage, les coupes de $5 \mu$ d'épaisseur, débarrassées du sublimé par le lugol, ont été colorées par le P.A.S. et l'hématoxyline de Harris modifiée. Jilles ont été ensuite recouvertes d'émulsion nucléaire pelliculable Kodak $A R$ so (Doniacir et P'ELC, 1950), exposées à $4^{\circ} \mathrm{C}$ pendant I à 3 mois, puis soumises aux processus de révélation photographique (FARAGGI, 1952).

Les stades du cycle de l'épithélium séminitère ont été définis en se basant sur le développement du système acrosomique des spermatides (ClerMon'T et IfEBLOND, 1952) et sur les différentes associations cellulaires (ROOSEN-RUNGe et (iIESEL, 1950). L'utilisation de ces critères a été rendue nécéssaire par la disparition des spermatides à noyau allongé consécutive à l'hypophysectomie.

Nous avons distingué les stades suivants :

Stade I : Déformation des spermatides : lcur noyau qui était rond devient ovale et bosselé. Près de la membrane basale les jeunes spermatocytes I sont au stade leptotène.

Stade 2 : Allongement des spermatides: elles deviennent fusiformes et leur noyau s'étire progressivement. Les jeunes spermatocytes I sont toujours au stade leptotène. Jes vieux spermatocytes I sont en fin de stade pachyténe (noyaux plus grands qu'au stade I).

Stade 3 : Les spermatides se rassemblent en faisceaux et leur noyau continue à s'étirer et à s'aplatir. Les jeunes spermatocytes I sont au stade zygotène. Les vieux spermatocytes I sont en fin de stade pachytène ou au stade diplotène. Chez les animaux hypophysectomisés, seule l'apparition des spermatocytes zygotène permet de séparer les stades 2 et 3 .

Stade 4 : C'est le stade des divisions de maturation : sont aussi classés au stade 4 tous les tubes où coexistent des spermatocytes I et II ou des spermatocytes II et des jeunes spermatides. 

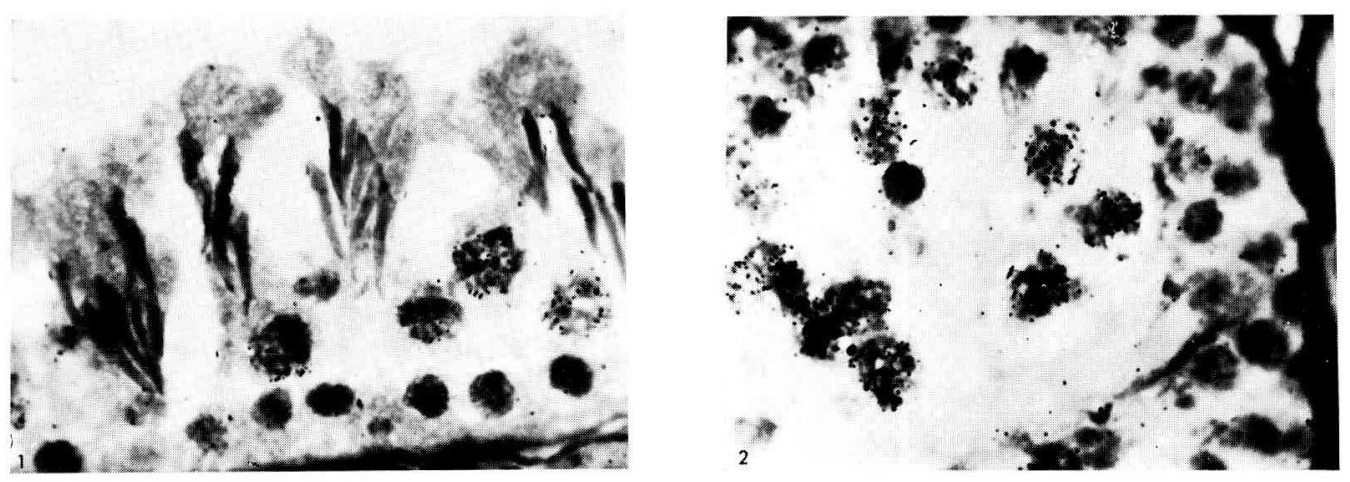

Fıs. I. -. Alutoraliographie d'un testicule de Rat normal avant recu une iniection de thymidine tritiée el étant abaltu 14,3 jours aprés. Les spermatocyles I au slade diplolène représentent la catégorie rellulaire marquée la plus arancée.

FIG. 2: - Autoradiographie dun testicule de Rat hypophysectomise, ayant resu une injection de thymidine tritiée 3 jours après l'hypophysectomie et étant aballu $\mathbf{1} 4,3$ jours plits tard. Les spermalocytes $I$ an stade diplotène représentent, comme che les animanx normatx, la calésonie cellulaire marquée la plits ancincée.
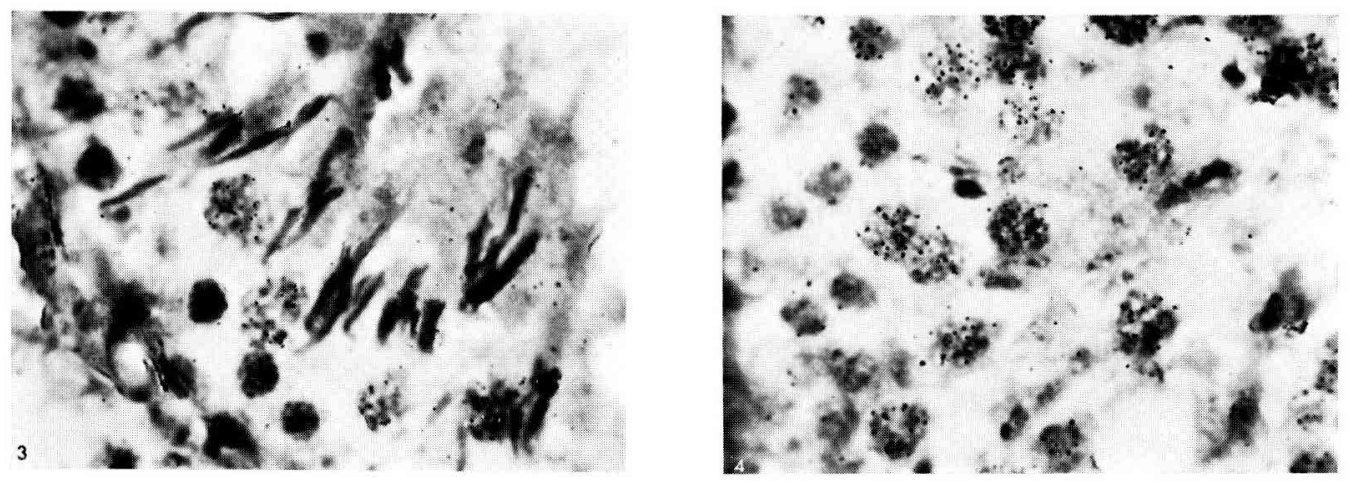

FIG. 3. -.. Alutoradiographie dun testicule de Rat hypophysectomisé, ayant regu une injection de thymidine tritiée 2 jours après lhypophysectonie puis injeclé biquotidiennement avec l'équivalent de i Io $\mu g$ de l.II standart Armour, et abith I4,3 jons après. Les calégories cellulaires marquées sont identiques antix précédentes.

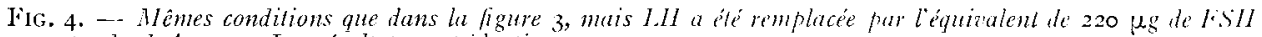
standard Arnour. Les résullats sont idenitiques 


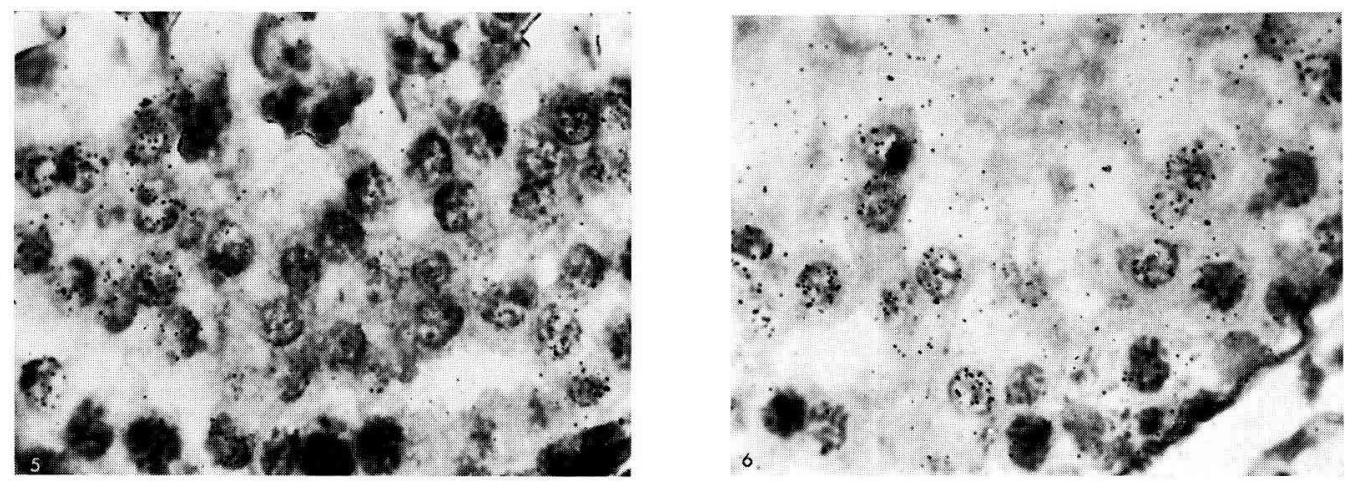

FIG. 5. -- Autoradiographie d'un testicule de Rat normal avant reçu une inieclion de thymidine tritiée et étant abattu 24,3 jours après. Les spermatides à noyau rond au stade 8 représentent la catégcrie cellulaire marquée la plus avancée.

Fig. 6. - Autoradiographie d'un testicule de Rat hypophysectomisé, avant reçu une injection de thymidine tritiee 2 jours après l'hypophysectomie et abattu 24,3 jours plus trird. Les catégories cellulaires marquées (spermatides) sont identiques à celles des animaux normaux.
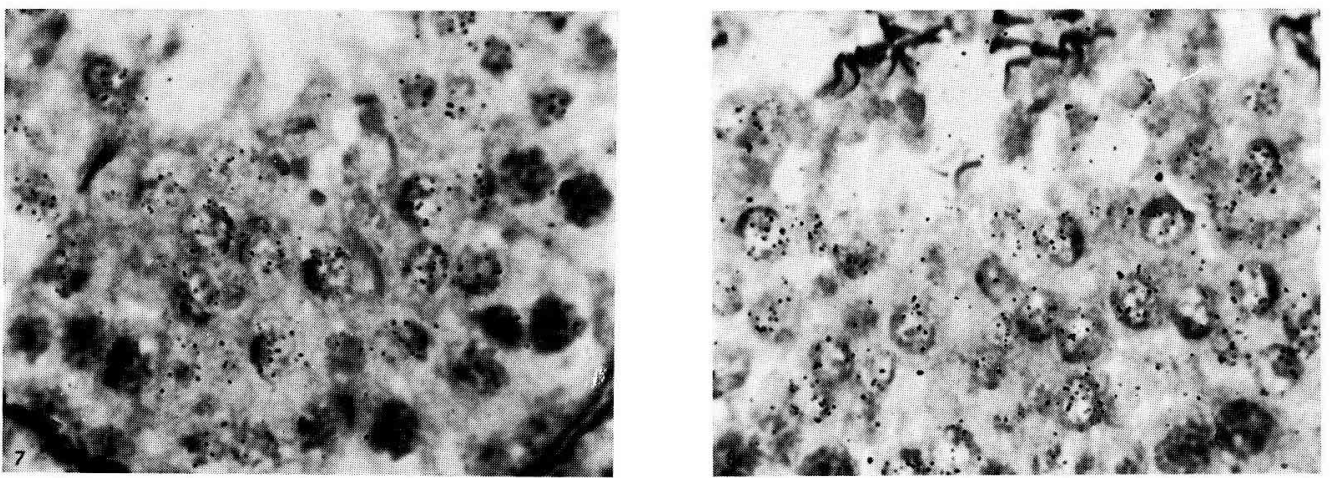

FIG. 7. - Auloradiographie d'un testicule de Rat hypophysectomisé, ayant recu une injection de thyntidine tritiée 2 jours après l'hypophysectomie puis injecté biquotidiennement avec l'équivalent de i o $\mu g$ de LH slandard Armour et abatu 24,3 jours après. Les catégories cellulaires marquées sont identiques à celles des figures 5 et 6 .

Fig. 8. - Mêmes conditions expérimentales que dans la figure 7 , mais 1 H a été remplacée par l'équizalent de $440 \mu g$ de FSH standard Amotir. Les résultats sont identiques 
Stade 5a : Présence d'une nouvelle génération de spermatides rondes. Leur idiosome ne contient pas de granule proacrosomique colorable par le P.A.S.

Stade 5b : Apparition d'un ou de plusieurs granules proacrosomiques qui fusionnent, par la suite, au niveau de l'idiosome des spermatides rondes. Le granule acrosomique n'est pas en contact avec la membrane nucléaire.

Stade 6 : Le granule acrosomique entre en contact avec la membrane nucléaire de la spermatide. Il s'aplatit et s'étale sur le noyau jusqu'à former, vu de profil, un arc de cercle recouvrant $1 / 6$ de la circonférence nucléaire.

Stade 7 : Le capuchon acrosomique recouvre de $\mathrm{I} / 4$ à $\mathrm{I} / 3 \mathrm{du}$ noyau.

Stade 8 : Le capuchon acrosomique recouvre la moitié du noyau de la spermatide ronde. Les tnes spermatocytes près de la membrane basale sont au stade préleptotène ; quelques-uns d'entre $\checkmark$ sont déjà au stade leptotène.

Chez les animaux hypophysectomisés, les stades 8, i et 2 sont difficiles à distinguer les uns des aucres. Au stade 8, il subsiste en général des spermatides typiques de ce stade, alors qu'au stade I elles ont disparu. La distinction entre stade I et 2 se base sur la plus grande taille des spermatocytes pachytène dans le stade le plus avancé.

Les fréquences relatives des différents stades du cycle de l'épithélium séminifère ont été établies de la manière suivante : après développement, les autoradiographies ont été photographiées à un grossissement de 30 fois environ. Chaque section transversale de tube séminifère observée au microscope a été repérée et son stade inscrit sur la photographie, ainsi que la présence ou l'absence de radioactivité. En aucun cas, les numérations n'ont porté sur moins de 500 tubes par animal. Cette technique donne des résultats analogues à ceux des méthodes décrites antérieurement. (ORTAVANT i958; Hochereau, Ig63).

Fn observant à quel stade du cycle de l'épithélium séminifère les cellules marquées les plus évoluées sont parvenues, on a pu déterminer et comparer les durées de ces stades et des différents processus spermatogénétiques.

Dans l'heure qui suit l'injection de thymidine tritiée, les cellules germinales les plus âgées qui incorporent ce précurseur de l'ADN sont les spermatocytes I au stade préleptotène (CLERMONT et a..., I959). Ces cellules devront encore évoluer pendant i 5 jours avant d'entreprendre leurs divisions de maturation. Par conséquent, si on prélève les testicules 14,3 jours après l'injection, on pourra vérifier, d'après la position des spermatocytes I marqués, si la durée de la prophase méiotique a varié avec les différentes conditions expérimentales.

De même, les premières spermatides marquées apparaissent environ $\mathbf{I}_{5}$ jours après l'injection de thymidine tritiée, et les spermatozoïdes sont libérés dans la lumière des tubes séminifères 36 jours après cette injection (CLERMont et al., I959). Pour savoir si la durée de la première partie de la spermiogenèse variait, nous avons effectué les prélèvements 24,3 jours après l'injection. $A$ ce moment, les spermatides marquées sont âgées de 9 jours environ. Des prélèvements plus tardifs n'ont pas été tentés, car les spermatides plus âgées (allongement du noyau) ne se forment pas après hypophysectomie.

Enfin, le niveau de production spermatogénétique a été calculé en appliquant la formule préconisée par Amann (1962) :

Nombre total de cellules germinales d'une catégorie dans le testicule $=$

Volume testicule $\times$ coeff. rétraction histologique $\times$ p. Ioo tubes sém.

$\times$ Nbre corrigé de cellules/section de tube

Surface de section de tube $x$ épaisseur section $\times$ durée cycle épithél. séminif.

Le coefficient de rétraction histologique a été estimé à 50 p. Ioo; la durée du cycle de l'épithélium séminifère considérée comme égale à I 2 jours selon CLERMONT et al. (I959).

\section{RÉSULTATS}

\section{Niveaux de production spermatogénétique}

Les résultats rapportés dans les tableaux I et 2 montrent que des équilibres endocriniens différents peuvent agir à trois niveaux dı cycle spermatogénétique : sur les divisions spermatogoniales, sur la prophase méiotique ou sur la spermiogenèse. 
TABLEAU I

Nombre $\left({ }_{1}{ }^{6}\right)$ de spermatocyles et de spermatides produits par jour dans un testicule de Rat (Hypophysectomie 16 jours avant ; durée du traitement: I4 jours ; doses exprimées en équivalent standard Armour par jour $\left(L H_{1}=\mathrm{I} 4 \mu \mathrm{g} ; L H_{2}=55 \mu \mathrm{g} ; L_{4}=220 \mu \mathrm{g} ; F S H_{1}=55 \mu \mathrm{g}\right.$; $\left.\mathrm{FSH}_{2}=220 \mu \mathrm{g} ; \mathrm{FSH}_{3}=440 \mu \mathrm{g} ; \mathrm{FSH}_{4}=\mathrm{I} 760 \mu \mathrm{g}\right) \mathrm{n}=$ nombre d'animaux

\begin{tabular}{|c|c|c|c|c|}
\hline Lot expérimental & Leptotène & Pachytène & Diplotène & $\begin{array}{c}\text { Jeunes } \\
\text { spermatides }\end{array}$ \\
\hline Contrôles & $10,32 \pm 1,18$ & $9,50 \pm 1,41$ & $9,50 \pm 0,91$ & $31,34 \pm 3,95$ \\
\hline$n=3$ & $8,38 \pm 1,43$ & $3,88 \pm 0,90$ & $2,56 \pm 0,27$ & $11,42 \pm 1,62$ \\
\hline$\overline{\mathbf{H}}+\mathrm{LH}_{\mathbf{I}}$ & $7,18 \pm 0,60$ & 5,58 上 0,31 & $3,14 \pm 0,39$ & $10,38 \pm 1,34$ \\
\hline$\overline{\mathbf{H}}+\mathrm{LH}_{2}$ & $8,34 \pm 0,88$ & 7,37 上 1,06 & $5,63 \pm 1,53$ & $18,42 \pm 3,07$ \\
\hline$\overline{\mathbf{H}}+\mathrm{LH}_{\mathfrak{u}}$ & $7,50 \pm 0,63$ & $7,28 \pm 0,60$ & $6,5^{\prime} \pm 1,96$ & $25,14 \pm 2,06$ \\
\hline$\overline{\overline{\mathbf{H}}}+\mathrm{FSH}_{1}$ & $7,48 \pm 0,17$ & $6,99 \pm 0,32$ & $4,75 \pm 0,75$ & $14,13 \pm 1,89$ \\
\hline$\overline{\overline{\mathbf{H}}+\mathrm{FSH}_{2}}$ & $9,06 \pm 0,77$ & $8,50 \pm 2,28$ & $8,78 \pm 1,02$ & $27,92 \pm 6,13$ \\
\hline$\overline{\mathbf{H}}+\mathrm{FSH}_{3}$ & $10,85 \pm 0,90$ & 9,63 上 0,57 & $9,55 \div 0,87$ & $32,8^{\prime} \pm 2,74$ \\
\hline$\overline{\overline{\mathrm{H}}}+\mathrm{FSH}_{4}$ & $12,66 \pm 1,97$ & $10,05 \pm 1,46$ & $9,71 \pm 1,51$ & $30,93 \pm 7,37$ \\
\hline$\overline{\overline{\mathbf{H}}}+\mathrm{FSH}_{4}+\mathrm{LH}_{4} \quad n=2$ & $10,6^{\prime}+1,28$ & $7,75 \pm 0,00$ & $8,50 \div 0,47$ & $27,73 \pm 1,50$ \\
\hline$\overline{\mathbf{H}}+\mathrm{FSH}_{2}+\mathrm{LH}_{1} \quad n=2$ & $8,01 \pm 1,09$ & $6,51 \pm 0,15$ & $4,95 \pm 0,35$ & $\overline{16,00 \pm 1,31}$ \\
\hline
\end{tabular}

Action sur les divisions spermatogoniales.

Cet effet est mesuré globalement par les variations du nombre de spermatocytes I au stade préleptotène produits quotidiennement. Bien que les multiplications spermatogoniales continuent après hypophysectomie, celle-ci diminue leur efficacité à $8 \mathrm{r}$ p. Ioo et $42 \mathrm{p}$. Ioo environ de leur niveau normal après I6 et 26 jours respectivement.

Par contre, l'injection d'hormone folliculostimulante à partir d'une dose quotidienne équivalant à $220 \mu \mathrm{g}$ du Standard Armour maintient cette efficacité à son niveau normal; de fortes doses de cette hormone ( $760 \mu \mathrm{g}$ de standard Armour) injectées quotidiennement semblent même améliorer l'efficacité des divisions spermatogoniales par rapport aux animaux normaux.

L'hormone interstitielle stimulante ne semble avoir qu'une faible action sur ces divisions; mais l'amélioration éventuelle de leur efficacité par des doses supérieures à celles employées ne peut être exclue.

Action sur la prophase méiotique.

Les différents traitements ont eu une action extrêmement importante sur cette fraction de la spermatogenèse. L'hypophysectomie entraîne une très sévère dégénérescence des spermatocytes $I$, surtout au cours des stades zygotène et pachytène. 
Ainsi, I6 et 26 jours après l'opération, il ne reste plus en fin de prophase méiotique (stade diplotène) que 33 p. Ioo et I3 p. roo des spermatocytes produits.

\section{TABLEAU 2}

Nombre $\left(\mathrm{I}^{\mathbf{6}}\right)$ de spermatocytes et de spermatides produits par jour dans un testicule de Rat (Hypophysectomie 26,3 jours avant; durée du traitement : 24 jours ; mêmes doses d'hormones que dans le tableau $\mathrm{r})$

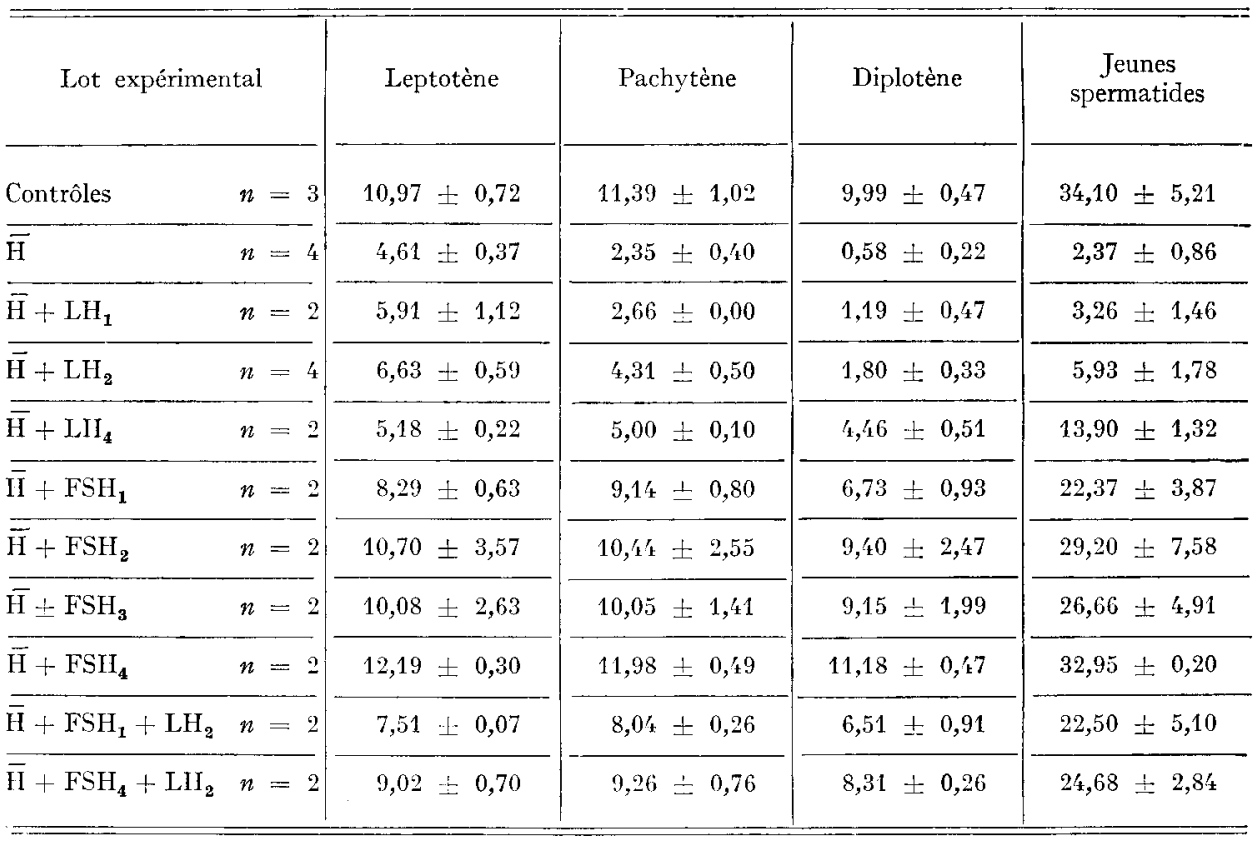

Par contre, les deux hormones gonadotropes utilisées préviennent ces phénomènes dégénératifs. Avec une dose équivalant à $220 \mu \mathrm{g}$ du standard L/H Armour et à 55 ou $220 \mu \mathrm{g}$ du standard FSH Armour, la prophase méiotique est pratiquement comparable à celle des animaux normaux : On obtient au stade diplotène 80 à 90 p. roo des spermatocytes dénombrés au stade leptotène. Pour des doses plus élevées, il ne semble pas se produire d'amélioration supplémentaire contrairement à ce qui se passe pour les multiplications spermatogoniales, et, pour des doses plus faibles, on obtient une réponse qui semble en rapport avec la dose. Fnfin, il ne semble pas exister de différence dans l'action des deux hormones FSH et ICSH sur cette partie du cycle spermatogénétique.

Action sur la spermiogenèse.

Chez les animaux hypophysectomisés, nous avons constaté la présence de quelques jeunes spermatides à l'intérieur des tubes séminifères. Leur nombre est approximativement 4 fois plus élevé que celui des spermatocytes I au stade diplotène. Les divisions de maturation ne semblent donc pas affectées par l'hypophy- 
sectomie tout au moins dans les 26 jours qui suivent l'opération. Ces résultats confirment ceux de Clermont et Morgenthaler (I955). Par contre, toutes les spermatides disparaissent lors de la phase d'allongement nucléaire qui constitue une étape infranchissable chez le Rat hypophysectomisé.

Ici encore, l'injection des hormones FSH et ICSH permet de franchir ce stade critique et d'accomplir la totalité de la spermiogenèse avec des doses équivalant à $55 \mu \mathrm{g}$ pour FSH et $220 \mu \mathrm{g}$ pour $\mathrm{L}_{\mathrm{H}} \mathrm{H}$ (tabl. 3).

TABLEAU 3

Rapport entre le nombre brut de spermatides (spides) à noyau allongé et celui de spermatides à noyau rond au stade 5 de l'épithélium séminifère 16,3 et 26,3 jours après l'hypophysectomie (H) avec ou sans traitement (mêmes conditions expérimentales que précédemment)

\begin{tabular}{|c|c|c|}
\hline \multirow{2}{*}{ Lot expérimental } & \multicolumn{2}{|c|}{ Spides noyau allonge/Spides noyau rond } \\
\hline & 16,3 jours après $\overline{\mathrm{H}}$ & 26,3 jours après $\overline{\mathrm{H}}$ \\
\hline Contrôles... & 0,75 & 0,76 \\
\hline$\overline{\mathrm{H}}$. & 0,00 & 0,00 \\
\hline$\overline{\mathrm{H}}+\mathrm{LH}_{1}$ & 0,39 & 0,00 \\
\hline$\overline{\mathrm{H}}+\mathrm{LH}_{2} \ldots \ldots \ldots \ldots$ & 0,39 & 0,00 \\
\hline$\overline{\bar{I}}+\mathrm{LH}_{4} \ldots \ldots \ldots \ldots$ & 0,77 & 0,45 \\
\hline$\underline{\overline{\mathrm{H}}}+\mathrm{FSH}_{1} \ldots \ldots \ldots$ & 0,81 & 0,68 \\
\hline$\underline{\overline{\mathrm{H}}}+\mathrm{FSH}_{2} \ldots \ldots \ldots$ & 0,70 & 0,81 \\
\hline$\overline{\mathrm{H}}+\mathrm{FSH}_{3} \ldots \ldots$ & 0,77 & 0,84 \\
\hline $\overrightarrow{\mathrm{H}}+\mathrm{FSH}_{4} \ldots \ldots \ldots$ & 0,77 & 0,75 \\
\hline$\overline{\mathrm{H}}+\mathrm{FSH}_{1}+\mathrm{LH}_{2} \ldots$ & 0,67 & 0,69 \\
\hline $\overrightarrow{\mathrm{II}}+\mathrm{FSH}_{2}+\mathrm{LH}_{2} \ldots$ & 0,64 & 0,75 \\
\hline
\end{tabular}

Ainsi, dans cette expérience, à l'aide de différents niveaux de supplémentation en hormones gonadotropes, il a été possible d'obtenir différents niveaux d'activité spermatogénétique, soit au cours de la prophase méiotique, soit au cours de la spermiogenèse. Examinons maintenant si la durée de ces processus spermatogénétiques a été modifiée.

\section{Durée des processus spermatogénétiques}

a) Durée de la prophase méiotique.

Dès l'injection chez l'animal normal, la thymidine tritiée est incorporée dans les noyaux des spermatocytes primaires au stade préleptotène.

I4,3 jours après l'injection les spermatocytes marqués les plus évolués sont au début du stade diplotène, c'est-à-dire à la fin du stade 2 et au début du stade 3 du cycle de l'épithélium séminifère. On peut définir la position des cellules marquées par la fraction de cycle comprise entre le début de celui-ci et le stade contenant les 
spermatocytes radioactifs. Cette fraction de cycle a été exprimée en p. Ioo du cycle complet de l'épithélium séminifère (ChERMONT et al., I959). Comme le montrent les résultats rapportés dans le tableau 4 la position des cellules marquées est pratiquement identique pour tous les lots considérés. Seul le lot d'animaux hypophysectomisés montre une progression légèrement accélérée; 1a différence n'est toutefois pas statistiquement significative, et peut résulter de la difficulté à identifier certains stades après hypophysectomie. (fig. I, 2, 3, 4).

\section{TABLEAU 4}

Position des cellules germinales marquées les plus avancées par rapport au début du cycle de l'épithélium séminifére; cette position est exprimée en pourcentage du cycle de l'épithélium (mêmes conditions expérimentales que dans les tableaux précédents)

\begin{tabular}{|c|c|c|}
\hline \multirow{2}{*}{ Lot expérimental } & \multicolumn{2}{|c|}{ Délai après l'injection de thymidine } \\
\hline & 14,3 jours & 24,3 jours \\
\hline Contrôles. & $+1 \%, 28 \pm 0,40$ & $89,10 \pm 0,22$ \\
\hline & $+19,60 \doteq 2,80$ & $95,52 \pm 0,32$ \\
\hline$\overline{\mathrm{H}}+\mathrm{LH}_{\mathbf{1}}$ & $+14,99$ 上 1,10 & \\
\hline$\overline{\mathrm{H}}+\mathrm{LH}_{2}$ & $+15,27 \pm 0,48$ & $89,58 \doteq 0,67$ \\
\hline$\overline{\mathrm{H}}+\mathrm{LH}_{4} \ldots \ldots \ldots \ldots$ & $+15,40 \pm 0,53$ & $89,60 \pm 1,27$ \\
\hline$\overline{\overline{\mathrm{H}}}+\mathrm{FSH}_{1} \ldots \ldots \ldots$. & $+14,41 \pm 0,93$ & \\
\hline$\underline{\overline{\mathrm{H}}}+\mathrm{FSH}_{2}$ & $+14,92 \pm 1,31$ & $85,08(1)$ \\
\hline$\overline{\mathrm{H}}+\mathrm{FSH}_{3}$. & $+16,22 \pm 0,37$ & $91,68(1)$ \\
\hline$\overline{\bar{H}}+\mathrm{FSIH}_{4}$ & $+15,32 \pm 0,49$ & $90,15 \pm 0,67$ \\
\hline$\underline{\overline{\mathrm{H}}}+\mathrm{FSH}_{3}+\mathrm{LH}_{2} \ldots$ & $+14,37 \pm 0,30$ & $89,74(1)$ \\
\hline$\overline{\mathrm{H}}+\mathrm{FSH}_{2}+\mathrm{LH}_{2} \ldots$ & $+16,97 \pm 1,58$ & $92,08 \pm 0,74$ \\
\hline
\end{tabular}

La fraction de cycle où sont localisées les cellules marquées représente la dispersion de ces cellules; elle est fonction du synchronisme de leur évolution. La dispersion des spermatocytes I marqués n'a été influencée par aucun des traitements (tabl. 5).

Ainsi, non seulement la vitesse de déroulement de la prophase méiotique n'a pas varié avec les conditions expérimentales, mais de plus toutes les cellules marquées ont évolué de façon pratiquement synchrone, quel qu'ait été le traitement.

\section{b) Durée de la spermiogenèse.}

Bien que, pour des raisons encore mal définies, quelques animaux aient donné des autoradiographies négatives, nous avons pu faire les constatations suivantes. Les spermatides marquées les plus âgées se rencontrent dans les tubes séminifères à la moitié du stade 8 du cycle de l'épithélium séminifère et ce dans tous les lots expérimentaux (tab1. 4). La dispersion de ces spermatides n'est pas modifiée à la 
suite des divers traitements, elle est la même que celle des spermatocytes I marqués I4,3 jours après injection de thymidine tritiée (tabl. 5).

Ainsi, pendant le début de la spermiogenèse, les spermatides évoluent toujours à la même vitesse et de façon synchrone, indépendamment du traitement, et quel que soit le niveau de production spermatogénétique (fig. $5,6,7,8$ ).

TABLEAU 5

Dispersion de la catégorie de cellules germinales marquées la plus avancée exprimée en pourcentage du cycle de l'épithélium séminifère

\begin{tabular}{|c|c|c|}
\hline \multirow{2}{*}{ Lot expérimental } & \multicolumn{2}{|c|}{ Délai après l'injection de thymidine } \\
\hline & 14,3 jours & $2^{2}, 3$ jours \\
\hline Contrôles.. & $11,63 \doteq 0,95$ & $12,73 \pm 0,50$ \\
\hline & $12,72 \pm 0,64$ & $16,08 \pm 0,60$ \\
\hline$\overline{\mathrm{H}}+\mathrm{LH}_{\mathbf{1}}$ & $11,05 \doteq 2,05$ & \\
\hline$\overline{\mathrm{H}}+\mathrm{LH}_{2}$ & $11,51 \pm 0,71$ & $11,24 \pm 0,33$ \\
\hline$\overline{\mathrm{II}}+\mathrm{LH}_{4}$ & $11,98 \doteq 0,10$ & $11,42 \pm 0,25$ \\
\hline $\overrightarrow{\mathrm{H}}+\mathrm{FSH}_{1} \ldots$. & $11,66 \pm 1,65$ & \\
\hline$\underline{\overline{\mathrm{H}}}+\mathrm{FSH}_{2} \ldots$. & $12,40 \pm 0,97$ & 9,68 \\
\hline$\overline{\overline{\mathrm{H}}}+\mathrm{FSH}_{3} \ldots \ldots$ & $11,74 \pm 1,16$ & 12,91 \\
\hline$\overline{\mathrm{H}}+\mathrm{FSH}_{4} \ldots$ & $11,90 \pm 0,86$ & $12,37 \pm 2,46$ \\
\hline$\overline{\mathrm{H}}+\mathrm{FSH}_{3}+\mathrm{LH}_{2} \ldots$ & $10,63 \pm 0,20$ & $14,65 \pm 0,44$ \\
\hline$\overline{\mathrm{I}}+\mathrm{FSH}_{2}+\mathrm{LH}_{2} \ldots$ & $14,13:=0,64$ & 11,82 \\
\hline
\end{tabular}

\section{DISCUSSION}

\section{Action des hormones gonadotropes}

Dans cette étude, nous avons utilisé des hormones gonadotropes peu purifiées. En effet, notre but était d'obtenir différents niveaux d'activité spermatogénétique plutôt que d'étudier l'action spécifique de ces hormones sur la spermatogenèse. Nos résultats permettent pourtant de dégager quelques indications à ce sujet.

Il est courant d'affirmer que les multiplications spermatogoniales échappent en grande partie à l'action des hormones hypophysaires. En réalité, nous constatons qu'il n'en est rien : après l'hypophysectomie, l'injection de doses quotidiennes suffisantes de FSH (220 $\mu \mathrm{g}$ de standard Armour) restaure l'efficacité des divisions spermatogoniales. La plus forte dose de FSH utilisée ( $1760 \mu \mathrm{g}$ de standard Armour) permet même d'obtenir une activité spermatogoniale supérieure à celle des animaux normaux, cependant la différence n'est pas significative. Cette tendance pourrait s'expliquer en partie par l'existence de phénomènes dégénératifs chez l'animal normal ( $C_{L}$ ERMONT, Ig62), phénomènes qui seraient prévenus par l'injection d'hormones gonadotropes. 
Mais l'action la plus spectaculaire s'exerce sans aucun doute sur la prophase méiotique et la spermiogenèse. Aussi bien FSH que LH empêchent les dégénérescences cellulaires pendant la spermatogenèse après hypophysectomie. Entre certaines limites, cette action semble même approximativement proportionnelle à la dose d'hormone utilisée.

\section{Durée des processus spermatogénétiques}

Dans aucun cas nous n'avons pu obtenir de variation de la durée, aussi bien de la prophase méiotique que de la première partie de la spermiogenèse. Bien plus, la dispersion des cellules marquées est restée pratiquement la même tout au long de la spermatogenèse. Ceci montre bien que les cellules germinales évoluent d'une façon synchrone et toujours à la même vitesse. Ceci est en contraste frappant avec la variabilité des niveaux d'activité spermatogénétique correspondants.

Ces observations sont en accord avec les résultats déjà obtenus chez le Bélier (ORTAVAN', I958) et chez le Rat (HARVEY et Cl.ERMONT, I962) dans d'autres conditions expérimentales. Ces derniers auteurs ont cependant montré que la durée de la spermatogenèse du Rat peut varier légèrement d'une souche à l'autre (HARVEY, I962). Les résultats quelque peu différents de MESs (I952) semblent dus à la difficulté éprouvée par cet auteur à identifier les stades du cycle spermatogénétique après hypophysectomie.

Chez le Rat, la spermatogenèse complète dure environ 48 jours (ClERMONT et al., I959) et s'amorce à la naissance. Nos résultats nous autorisent à penser que l'injection d'hormones gonadotropes après la naissance ne peut induire l'apparition prématurée de spermatozoïdes dans le testicule chez cette espèce, contrairement à ce qui a été rapporté (WAKELING, I959).

En conclusion, soulignons que la stimulation ou l'inhibition de la spermatogenèse n'impliquent aucunement son accélération ou son ralentissement.

Les hormones gamétocinétiques n'accélèrent pas la vitesse d'évolution des cellules germinales; elles permettent seulement à un plus grand nombre d'entre elles d'arriver à maturité.

Rę̧u pour publication en juillet I963.

\section{SUMMARY}

THE INFLUENCE OF GONADOTROPIC IIORMONES ON THE DURATION OF SPERMATOGENIC PROCESSES IN THE RAT

The influence of hypophysectomy and of treatment with gonadotropic hormones on the duration of the meiotic prophase and of spermiogenesis has been studied in 62 adult male rats.

Normal, hypophysectomized, and hypophysectomized-gonadotropin treated rats were injected a single dose of tritiated thymidin (0.8-1.ouc/g. body weight intraperitoneally, and 48 hours after the operation) in order to label the germ cells. These were studied by means of autoradiography. Gonadotropin treatment began at the time of thymidin injection and lasted I 4 or 24 days. Different dosages of FSH and LH were used, alone or in combinations (FSH : from $55 \mu \mathrm{g}$ to I $760 \mu \mathrm{g} / \mathrm{day}$ of Armours' Standard ; LH : from $14 \mu \mathrm{g}$ to $220 \mu \mathrm{g} /$ day of Armour's Standard.)

Hypophysectomy brings about an important degeneration of the germ cells. I6 days after hypophysectomy, no more than $33 \mathrm{p}$. roo of the primary spermatocytes at the diplotene stage and 
$36 \mathrm{p}$. Ioo of the spermatids with a round nucleus are produced, as compared with the controls. 26 days after hypophysectomy, these values are still lower : I 3 p. Ioo and 7 p. Ioo respectively. Spermatids with elongated nuclei are no longer present.

Although graded dosages of gonadotropins can inhibit germ cells degeneration and induce different corresponding levels of spermatogenic activity, none of these treatments is able to influence the speed of evolution and the synchronization of development of those cells which do not degenerate after hypophysectomy.

\section{RÉFÉRENCES BIBI,IOGRAPHIQUES}

Amann R. P., Almquist J. O., 1962. Reproduction capacity of dairy bulls. VIII Direct and indirect measurement of testicular sperm production. J. Dairy Sci., 45, 774-8I.

Clermont Y., I962. Quantitative analysis of spermatogenesis of the rat : a revised model for the renewal of spermatogonia. Amer. J. Anat., 111, i I I-29.

Clermont Y., Leblond C. P., Messier B., i959. Durée du cycle de l'épithélium séminal du Rat. Arch. Anat. Micros. Morph. Exper., $\mathbf{4 8}$ bis, 37-56.

Clermont Y., Morgenthaler H., 1955. Quantitative study of spermatogenesis in the hypophysectomized rat. Endocrinology, 57, 369-82.

Doniach I., Pelc S. R., I950. Autoradiograph technique. Brit. J. Radiol., 23, I84-92.

FARAGGI H., I952. Détection des éléments radioactifs par la technique photographique. Autoradiographie. Comm. En. Atom., France, 33 p.

GaArenstroom J. H., De Jongh S. E., i946. A contribution to the knowledge of the influences of ganodotropic and sex hormones on the gonads of rats. Monogr. Progr. Res. Holland during the War. Elsevier publ. Co Inc. New York, Amsterdam.

Greep R. O., Fevold H. L., Hisaw F. L., I936. Effects of two hypophyseal gonadotropic hormones on the reproductive system of the male rat. Anat. Rec., 65, 26r-72.

Harvey C., 1962. Duration of the cycle of the seminiferous epithelium in normal, hypophysectomized and hormone treated rats. M.Sci. Thesis, Mc Gill University.

Harvey C., Clermont Y., i962. The duration of the cycle of the seminiferous epithelium of normal, hypophysectomized and hypophysectomized hormone-treated albino rats. Anat. Rec., 142, 239-4o.

Heller C. G., Clermont Y., I963. Spermatogenesis in man. An estimate of its duration. Science, 14., I $84-95$.

Hochereau M. T., I963. Étude comparée de la vague spermatogénétique chez le Taureau et chez le Rat. Ann. Biol. anim. Bioch. Biophys., 3 (sous presse).

Leblond C. P., Clermont Y., 1952. Definition of the stages of the cycle of the seminiferous epithelium in the Rat. Ann. N. Y. Acad. Sci., 55, 548-73.

MEss B., I952. Influence of hypothalamic injury on spermatogenesis in albino rats. Acta Morph. Acad. Sci. Hung., 2, 275-85.

Ortavant R., I958. Le cycle spermatogénétique chez le Bélier. Thèse Doct. Sci. Paris, i 27 p.

Ortavant R., Orgebin M. C., Singh G., i $96 \mathrm{r}$. Étude comparative de la durée des phénomènes spermatogénétiques chez les animaux domestiques. Symposium on the use of radioisotopes in Animal Husbandry and Medical Sciences, Mexico City, 321-27.

PARLow A. F., 1958. A rapid bioassay method for LH and factors stimulating LH secretion. Feder. Proc., 19, 402 .

Pelletier J., r963: Étude de l'hormone stimulant l'interstitielle méthode de dosage et variation de la teneur hypophysaire du Bélier soumis à différentes durées d'éclairement. Thèse Doct. $3^{\mathrm{e}}$ cycle, Paris, $49 \mathrm{p}$.

Randolph P. W., Lostroh A. J., Grattarola R., Squire P. G., LI C. H., 1959. Effect of ovine interstitial cell-stimulating hormone on spermatogenesis in the hypophysectomized mouse. Endocrinology, 65, 433-41.

Roosen-Runge E. C., Giesel L. O., I950. Quantitative studies on spermatogenesis in the albino Rat. Amer. J. Anat., 8\%, 1-30.

Simpson M. E., Evans H. M., I946. Comparison of the spermatogenic and androgenic properties of testo sterone propionate with those of pituitary ICSH in hypophysectomized 40 day old male rats. Endocrinology, 39, 281-285.

SimPson M. E., Li C. H., Evans H. M., I95r. Synergism between pituitary follicle-stimulating hormone (FSH) and human chorionic gonadotropin (HCG). Endocrinology, 48, 370-383.

Steelman S. L., Pohley F. P., i 953. Assay of the follicle stimulating hormone based on the augmentation with human chorionic gonadotrophin. Endocrinology, 53, 604-6r6.

WAKELING A., 1959. The effect of gonadotrophins and androgen on spermiogenesis in immature rat. J.Endocrin., 19, $263^{-273}$.

Woops M. C., Simpson M. E., I96r. Pituitary control of the testis of the hypophysectomized rat. Endocrinology, 69, 9I-I 25 . 